\title{
Flaxseed oil and a-lipoic acid combination reduces atherosclerosis risk factors in rats fed a high-fat diet
}

\author{
Jiqu Xu ${ }^{1,2}$, Wei Yang ${ }^{3}$, Qianchun Deng ${ }^{1,2}$, Qingde Huang ${ }^{1,2}$, Jin'e Yang ${ }^{1,2}$ and Fenghong Huang ${ }^{1,2^{*}}$
}

\begin{abstract}
Background: Atherosclerosis is a major manifestation of the pathophysiology underlying cardiovascular disease. Flaxseed oil (FO) and a-lipoic acid ( $L A$ ) have been reported to exert potential benefit to cardiovascular system. This study tried to assess the effect of supplement of FO and LA combination on the atherosclerosis risk factors in rats fed a high-fat diet.

Methods: LA was dissolved in flaxseed oil to a final concentration of $8 \mathrm{~g} / \mathrm{kg}(\mathrm{FO}+\mathrm{LA})$ when used. The rodent diet contained $20 \%$ fat. One-fifth of the fat was soybean oil and the others were lard (HFD group), or $75 \%$ lard and 25\% $\mathrm{FO}+\mathrm{LA}$ (L-FO+LA group), or 50\% lard and 50\% FO+LA (M-FO+LA group), or FO+LA (H-FO+LA group). Animals were fed for 10 weeks and then killed for blood collection.

Results: Supplement of FO and LA combination significantly enhanced plasma antioxidant defense capacities, as evaluated by the marked increase in the activities of SOD, CAT and GPx as well as the level of GSH, and the significant reduction in lipid peroxidation. Simultaneous intake of FO and $L A$ also reduced plasma TG, TC and LDL-C contents and elevated the ratio of HDL-C/LDL-C. Besides, in parallel with the increase of FO and LA combination, plasma IL-6 and CRP levels were remarkably reduced.
\end{abstract}

Conclusion: Supplement of FO and LA combination may contribute to prevent atherogenesis by improving plasma oxidative stress, lipid profile and inflammation.

Keywords: Flaxseed oil, a-lipoic acid, Atherosclerosis, Oxidant stress, Plasma lipids, Inflammation

\section{Introduction}

Cardiovascular disease (CVD) is widely recognized as the leading cause of premature death and a major cause of disability in most developed and developing countries. Atherosclerosis, a manifestation of the pathophysiology underlying CVD, constitutes the single most important contributor to this growing burden of this disease. Recent studies suggest that oxidant stress [1], lipid abnormalities [2] and chronic inflammation [3] play important roles in the etiology of atherosclerosis and subsequent CVD.

\footnotetext{
* Correspondence: fhhuang@foxmail.com

${ }^{1}$ Department of Product Processing and Nutriology, Oil Crops Research Institute, Chinese Academy of Agricultural Sciences, 2 Xudong Second Road, Wuhan 430062, P.R. China

${ }^{2}$ Hubei Key Laboratory of Lipid Chemistry and Nutrition, Oil Crop s Research Institute, Chinese Academy of Agricultural Sciences, 2 Xudong Second Road, Wuhan 430062, P.R. China

Full list of author information is available at the end of the article
}

Flaxseed oil (FO) is the main component of the flaxseed and one of the world's most important vegetable sources of $\alpha$-linolenic acid (LNA, 18:3n-3). As a nutritionally essential polyunsaturated fatty acid (PUFA), LNA can act as the precursor of longer chain n-3 PUFA (EPA and DHA) or compete with linoleic acid or direct interaction with ion channels and nuclear receptors, and thus may exert various biological functions in the human body, such as accelerating brain growth in preterm and neonatesand, antiarrhythmic functions and neuroprotective functions [4]. In addition, LNA is also reported to have beneficial effects on blood lipid profiles [4-7] and inflammation $[4,8,9]$, which may responsible for the protection against CVD bestowed by FO. However, on the other hand, since LNA is highly susceptible to oxidation, FO addition leads to a significantly higher tendency toward plasma lipid peroxidation $[10,11]$, which may

\section{Biomed Central}


have an adverse effect on the protection of cardiovascular system.

$\alpha$-lipoic acid (LA), also referred to as thioctic acid, is a disulfide compound that is found naturally in mitochondria as the coenzyme for pyruvate dehydrogenase and $\alpha$-ketoglutarate thus serves a critical role in mitochondrial energy metabolism. Although orally supplied LA may not be used as a metabolic cofactor, there are a unique set of biochemical activities with potential pharmacotherapeutic value against a host of pathophysiologic insults [12]. For example, LA has gained considerable attention as an excellent antioxidant to reduce oxidative stress [13-15]. Further, LA is fat- and water-soluble, which makes it effective against a broader range of free radicals. Early studies have shown the capacity of LA to decrease plasma lipids in rats [16-18]. Besides, LA also exhibits an anti-inflammatory activity in clinical trial [19]. These mechanisms make LA possess the potential abilities for antiatherogenesis.

To our knowledge, the effects of a simultaneous intake of FO and LA on cardiovascular system have not been investigated. Therefore, the objectives of this study were to determine that whether FO and LA combination can reduce atherosclerosis risk factors in rats fed a high-fat diet.

\section{Materials and methods}

\section{Chemical sources}

Commercial deodorized lard was purchased from a local supermarket. The flaxseed oil was obtained from Caoyuankangshen Food Co., Ltd., and its fatty acid compositions were listed in Table 1. LA was purchased from Sigma-Aldrich (St. Louis, MO, USA) and was dissolved in flaxseed oil to a final concentration of $8 \mathrm{~g} / \mathrm{kg}(\mathrm{FO}+\mathrm{LA})$ when used.

\section{Animals and diets}

The experiment was conducted with 40 male SpragueDawley rats (initially weighing 150-170 g). The animals were housed individually and maintained at a controlled ambient temperature $\left(24 \pm 1{ }^{\circ} \mathrm{C}\right)$ under diurnal conditions (light-dark: 08:00-20:00) with access to laboratory chow and tap water ad libitum. After 1 week of acclimatization, rats were randomly divided into a high-fat diet (HFD) group and three experimental groups ( $\mathrm{n}=10$ per group).

Table 1 Fatty acid compositions of flaxseed oil

\begin{tabular}{lc}
\hline Fatty acid & Composition (wt.\%) \\
\hline Palmitic acid (C16:0) & 6.1 \\
Stearic acid (C18:0) & 3.7 \\
Oleic acid (C18:1) & 22.4 \\
Linoleic acid (C18:2) & 14.9 \\
Linolenic acid (C18:3) & 52.9 \\
\hline
\end{tabular}

All animals were fed purified experimental diets which contained 35\% maize starch, 20\% casein, 15\% sucrose, 5\% cellulose, $3.5 \%$ mineral mixture (AIN-93M), $1 \%$ vitamin mixture (AIN-93M), 0.3\% DL-methionine, 0.2\% choline bitartrate and $20 \%$ fat. One-fifth of the fat in the diet of each group was soybean oil and the others were lard (HFD group), or $75 \%$ lard and 25\% FO+LA (L-FO+LA group), or $50 \%$ lard and 50\% FO+LA (M-FO+LA group), or $\mathrm{FO}+\mathrm{LA}$ (H-FO+LA group). Every week, all ingredients for the purified diets were mixed, formed into a dough with purified water, rolled into pellets, sealed in air-tight plastic bags under nitrogen gas and stored at $-80^{\circ} \mathrm{C}$ until use. The food in the animal cages was shaded from light and changed every day. The animals were cared for in accordance with the Guiding Principles in the Care and Use of Animals. The experiment was approved by the local animal care committee.

\section{Blood processing}

After 10 weeks of feeding, animals, fasted for 16 hours, were killed under anaesthesia, blood was collected in the presence of sodium heparin from the heart immediately. Blood samples were centrifuged at $1500 \mathrm{~g}$ for $10 \mathrm{~min}$ at $4^{\circ} \mathrm{C}$ and the plasma was stored at $-80^{\circ} \mathrm{C}$ until analysis.

\section{Plasma lipids analysis}

The plasma triglyeride (TG), total cholesterol (TC), lowdensity lipoprotein cholesterol (LDL-C) and high-density lipoprotein cholesterol (HDL-C) levels were measured using commercial enzymatic kits (Zhongsheng Beikong Biotech Company, China).

Assay of liver antioxidant capacity and lipid peroxidation Superoxide dismutases (SOD) activity was measured according to the method of Kono [20]. Catalase (CAT) activity was estimated basing on the method of Goth [21]. Glutathione peroxidase (GPx) activity was measured by the method of Sazuka [22]. The glutathione (GSH) content was determined by the method of Moron [23]. The total antioxidant capability (T-AOC) was assayed with commercial kits (Nanjing Jiancheng Bioengineering Institute, China).Thiobarbituric acid reactive substances (TBARS) level was estimated by the method of Buege [24]. The detection procedure of these enzymes activities has been described in detail in our previous report [25].

\section{Assay of protein concentration}

The protein concentration was determined by the method of Lowry [26] with bovine serum albumin as the standard.

\section{Assay of plasma inflammatory markers}

The C-reactive protein (CRP) and interleukin 6 (IL-6) levels in plasma were measured with the use of 
commercially available rat CRP ELISA kit (eBioscience San Diego, CA) and rat IL-6 ELISA kit (eBioscience San Diego, CA), respectively. All the procedures and conditions were consistent with the instructions of these kits.

\section{Statistical analyses}

Values are presented as mean \pm SEM (standard error of the mean). The data were analyzed by one-way ANOVA, followed by the Fisher PLSD post hoc test if the overall differences were significant $(p<0.05)$. All statistical analyses were performed using SPSS 13.0 statistical software (SPSS Inc., Chicago, IL) and a difference was considered significant when $p<0.05$.

\section{Results}

Plasma antioxidative capacity and lipid peroxidation

As shown in Figure 1, animals in $\mathrm{M}$ - and $\mathrm{H}-\mathrm{FO}+\mathrm{LA}$ groups revealed significantly higher plasma antioxidant enzymes SOD, CAT and GPx activities than their counterparts in HFD group. Administration of L-, M- and $\mathrm{H}$ - FO+LA for 10 weeks significantly augmented the GSH levels in plasma when compared to rats treated with HFD. As the overall antioxidative capacity, plasma T-AOC in all three FO+LA groups was markedly higher than that in HFD group. Moreover, when plasma TBARS were evaluated as the marker of lipid peroxidation, there were significant decline in the levels of plasma
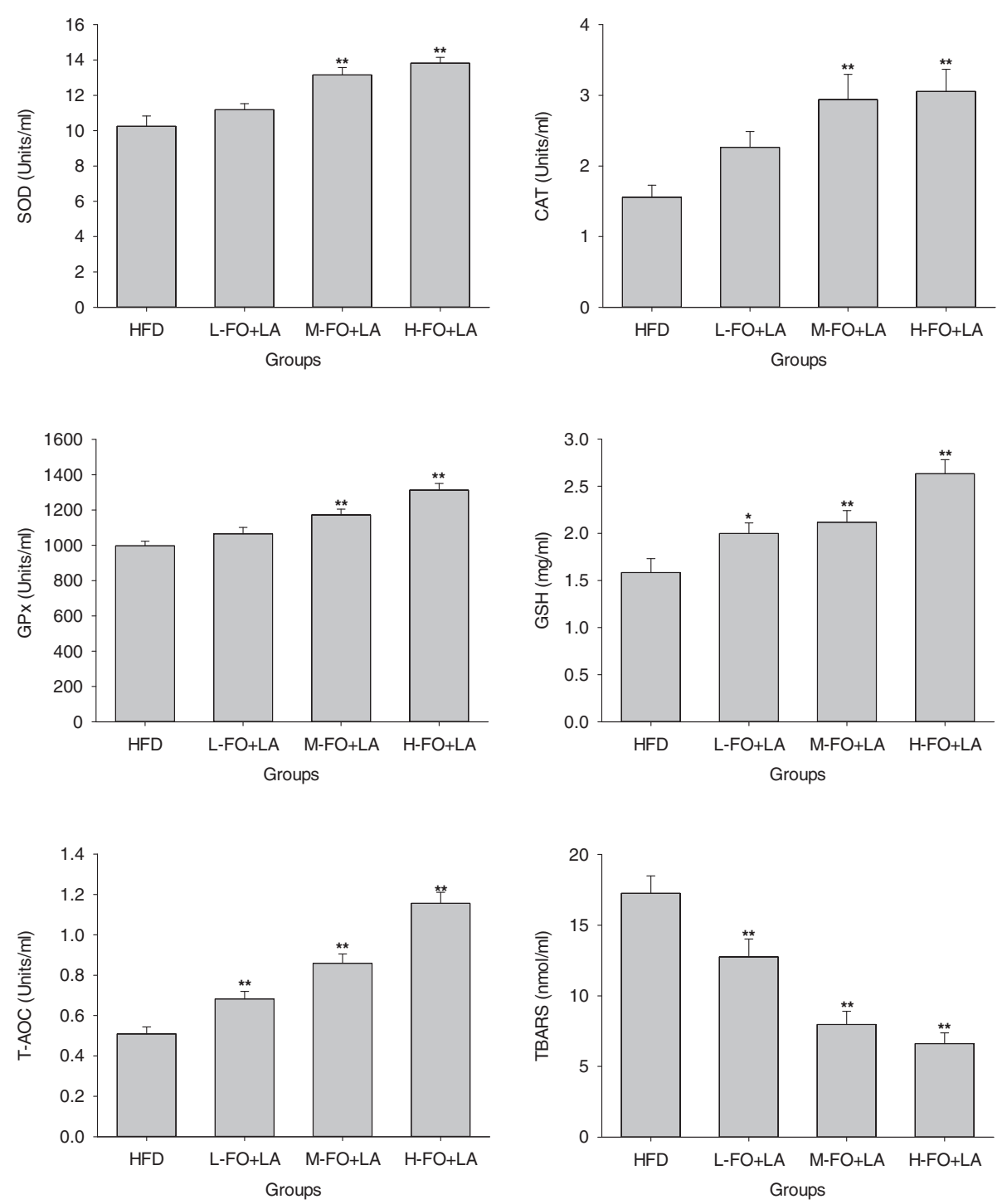

Figure 1 Effects of FO and LA combination on the activities of antioxidant enzymes (SOD, CAT and GPx), the levels of GSH, T-AOC and contents of TBARS in plasma of rats fed a high-fat diet. HFD: high-fat diet group; L-. M- and H- FO + LA: low, middle and high contents of FO and LA combination groups. Bars represent the mean \pm SD from 10 animals in each group. ${ }^{*} p<0.05$ and ${ }^{* *} p<0.01$ compared to the HFD group. 
TBARS in all three FO+LA groups when compared with those in HFD group.

\section{Plasma lipids}

The effects of FO+LA on the levels of plasma lipids are shown in Figure 2. Animals in M- and $\mathrm{H}$-FO+LA groups exhibited significantly lower plasma TG and TC levels than that in HFD group. There were no marked differences in the level of plasma HDL-C in all groups, while rats in all FO+LA groups showed significantly lower LDL-C levels than that in HFD group. As results, M- and $\mathrm{H}-\mathrm{FO}+\mathrm{LA}$ groups had significantly higher ratios of HDL to LDL cholesterol than HFD group.

\section{Plasma inflammatory}

As can be seen in Figure 3, there were significantly decline in the plasma levels of IL-6 and CRP in all three FO+LA groups when compared with those in HFD group.

\section{Discussion}

FO has gained more and more attention in many areas recently primarily because it is one of the richest sources of LNA. As the plant precursor of EPA and DHA, ALA may produce significant cardiovascular benefits which have been proved by some clinical trials, epidemiological investigations and experimental studies [27]. LA is a potent natural antioxidant and represents a possible
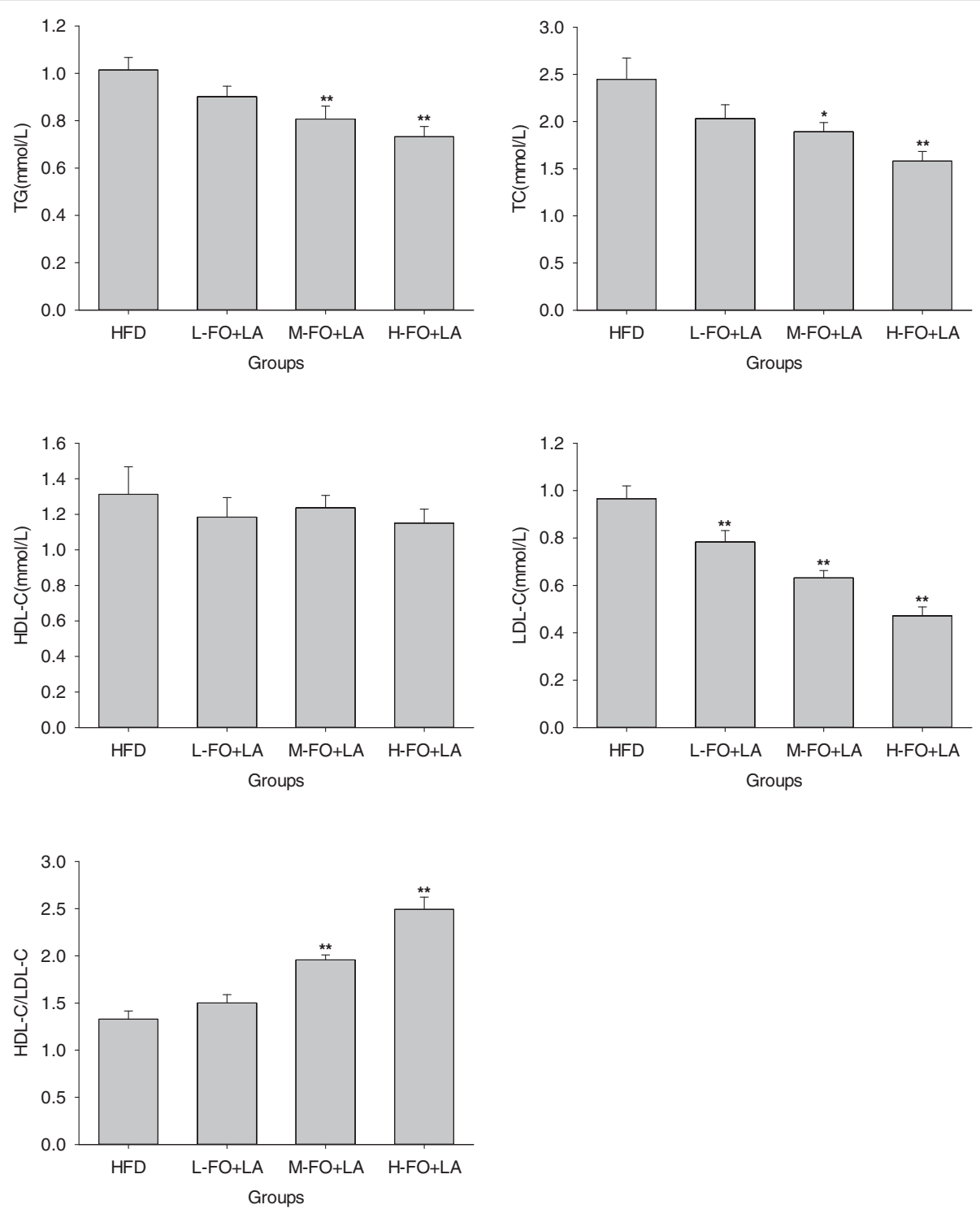

Figure 2 Effects of FO and LA combination on various plasma lipid parameters (TG, TC, LDL-C and HDL-C) and on the ratios of plasma HDL-C/LDL-C of rats fed a high-fat diet. HFD: high-fat diet group; L-. M- and H- FO + LA: low, middle and high contents of FO and LA combination groups. Bars represent the mean \pm SD from 10 animals in each group. ${ }^{*} p<0.05$ and ${ }^{* *} p<0.01$ compared to the HFD group. 

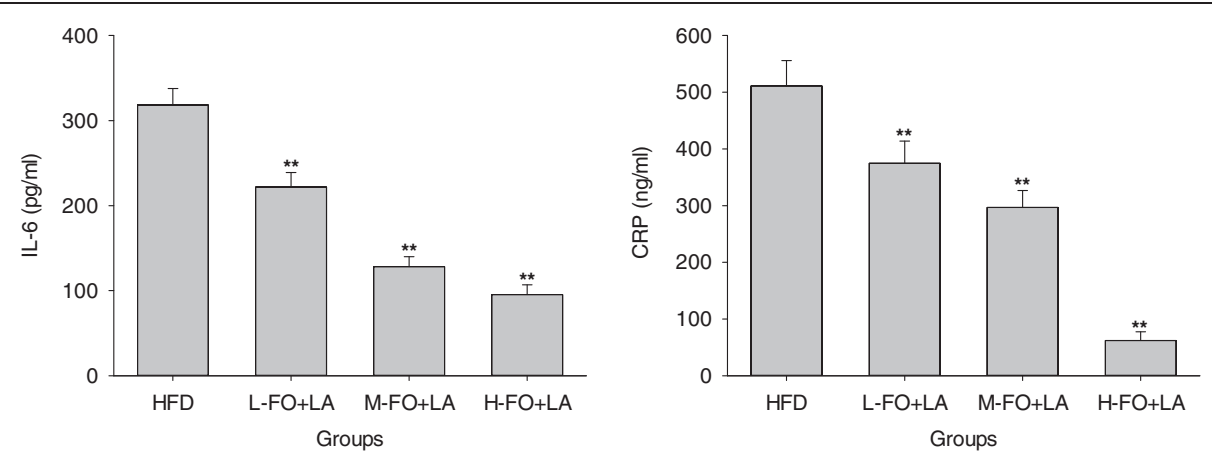

Figure 3 Effects of FO and LA combination on the level of IL-6 and CRP in plasma of rats fed a high-fat diet. HFD: high-fat diet group; L-. $\mathrm{M}$ - and $\mathrm{H}-\mathrm{FO}+\mathrm{LA}$ : low, middle and high contents of FO and LA combination groups. Bars represent the mean \pm SD from 10 animals in each group. ${ }^{* *} p<0.01$ compared to the HFD group.

protective agent against risk factors of CVD [28]. In this report, we examined the effect of FO and LA combination on atherosclerosis risk factors in rats fed a high-fat diet.

The imbalance between cellular production of free radicals and the antioxidant defense is referred to as oxidative stress. The relative excessive production of free radicals can attack all types of biomolecules, which may affect oxidation of low density lipoproteins and lead to the destruction of cellular components and thus initiates the processes of atherogenesis. Growing evidence indicates that oxidative stress is the pivotal pathogenetic factor and unifying mechanism for atherosclerosis and other cardiovascular diseases [29]. For example, free radicals are key mediators of various signaling pathways which underlie vascular inflammation in atherogenesis [30]. However, an efficient antioxidant defense mechanism (enzymatic and non-enzymatic) is able to counteract the deleterious effects of oxidative stress. The primary antioxidant enzymes in mammals include SOD which converts superoxide to hydrogen peroxide, GPx and CAT which are responsible for converting hydrogen peroxide to water [31]. GSH is a very important nonenzymatic antioxidant which can react directly with free radicals or act as an electron donor in the reduction of peroxides catalyzed by GPx [32]. In the present study, FO and LA combination markedly increased the plasma activities of these antioxidant enzymes (SOD, CAT and GPx) as well as GSH level, which resulted in pronounced enhancement of plasma T-AOC. As a consequence, plasma lipid peroxidation levels significantly declined with the supplement of FO and LA combination. However, FO itself is hardly thought to have any antioxidative activity and further, it may cause lipid peroxidation because of its susceptibility to oxidation $[10,11]$. Therefore, LA should impart the entire antioxidative potency in this experiment. In fact, LA has been proved to be a potent multifunctional biological antioxidant. It can reduce oxidative stress by scavenging free radicals, chelating transition metals, increasing intracellular GSH and the redox regeneration of other antioxidants such as vitamins C and E [13-15,33,34]. In addition, LA also decreases oxidative stress by restoring enzymatic antioxidant system in blood in different status $[34,35]$.

It is well known that hyperlipidemia is a highly predisposing condition for arteriosclerosis and other cardiovascular disease. A large body of evidence has been presented showing that high-fat diet rich in saturated fatty acid results in hyperlipidemia. In the present study, FO and LA combination decreased the plasma TG, TC and LDL-C levels, and both of them contributed to these beneficial changes. LNA and LA have both been reported to suppress the expression and activities of many hepatic fatty acid syntheses such as fatty acid synthase (FAS), malic enzyme and glucose 6-phosphate dehydrogenase [36-38], and hence decrease fatty acid synthesis in liver. On the other hand, LNA sharply enhances hepatic peroxisomal and mitochondrial fatty acid oxidation rate by increasing the expression and activities of a series of fatty acid oxidation enzymes [37,39], and LA also shows the similar action on inducing fatty acid oxidation [18]. In addition, FO has been shown to have hypocholesterolaemic effect, which might result from increases of hepatic LDL-receptor expression and cholesterol catabolism/output [7]. Administration of LA can likewise reduce the elevated plasma TC and LDL-C induced by high-fat diet by downregulating the expression of genes involved in cholesterol synthesis in liver [18]. Although simultaneous intake of FO and LA did not markedly affect plasma HDL-C level in the present study, the significant increase in the ratio of HDL to LDL cholesterol still meant that the combination of FO and LA had a marked protective effect with respect to atherosclerosis.

Based on strong experimental and clinical evidence, the general consensus is that inflammation plays a central role in both initiation and progression of the 
atherosclerotic process [40-42]. Various proinflammatory risk factors such as oxidized LDL and infectious agents can trigger the production of proinflammatory cytokines which are central mediators of inflammation associated with atherogenesis. As two of the most important proinflammatory cytokines, IL- 6 and CRP are associated with CVD and served as inflammatory markers for prediction of atherosclerotic risk [43-45]. In the present study, supplement of FO and LA remarkably reduced the plasma levels of both IL- 6 and CRP, which meant that combination of FO and LA has an ability to improve inflammation status. Supporting our results, intakes of dietary FO or LA have been shown to elicit antiinflammatory effects by inhibiting inflammatory cytokine production. For example, FO can suppress the expression of inflammatory cytokine such as IL-6, IL-1, CRP and TNF- $\alpha$ via a reduction in NF- $\mathrm{kB}$ induced gene expression and/or an activation of PPAR- $\gamma[4,8,46]$. Similarly, LA has also been demonstrated to inhibit NF- $\mathrm{KB}$ activation independent of its antioxidant function $[47,48]$ and thus reduce circulating levels of inflammatory markers [19].

In summary, the combination of FO and LA is effective in amelioration of oxidative stress, lipid profile and inflammation of plasma in rats fed a high-fat diet. These results suggested that supplement of FO and LA combination might contribute to prevent atherogenesis and then decrease the incidence of CVD.

\section{Competing interest}

No competing financial interests exist.

\section{Authors' contributions}

Author JX designed and wrote a first draft of the paper. WY, QD and JY carried out all the experiments. QH performed the data analysis and created the figures. FH contributed to the design of the study, reviewed the manuscript and contributed to the final version. All authors contributed to and have approved the final manuscript.

\section{Acknowledgments}

This work was supported by National Natural Science Foundation of China (NSFC-31171681) and the earmarked fund for Modern Agro-industry Technology Research System (CARS-17), china.

\section{Author details}

'Department of Product Processing and Nutriology, Oil Crops Research Institute, Chinese Academy of Agricultural Sciences, 2 Xudong Second Road, Wuhan 430062, P.R. China. ${ }^{2}$ Hubei Key Laboratory of Lipid Chemistry and Nutrition, Oil Crop s Research Institute, Chinese Academy of Agricultural Sciences, 2 Xudong Second Road, Wuhan 430062, P.R. China. ${ }^{3}$ Department of Nutrition and Food Hygiene, School of Public Health, Tongji Medical College, Huazhong University of Science and Technology, 13 Hangkong Road, Wuhan 430030, P.R. China

Received: 5 July 2012 Accepted: 26 October 2012

Published: 31 October 2012

\section{References}

1. Yokoyama M: Oxidant stress and atherosclerosis. Curr Opin Pharmacol 2004, 4(2):110-115.

2. Castelli WP, Garrison RJ, Wilson PW, Abbott RD, Kalousdian S, Kannel WB: Incidence of coronary heart disease and lipoprotein cholesterol levels. The Framingham Study. JAMA 1986, 256(20):2835-2838.
3. Hansson GK: Inflammation, atherosclerosis, and coronary artery disease. N Engl J Med 2005, 352(16):1685-1695.

4. Barcelo-Coblijn G, Murphy EJ: Alpha-linolenic acid and its conversion to longer chain n-3 fatty acids: benefits for human health and a role in maintaining tissue n-3 fatty acid levels. Prog Lipid Res 2009, 48(6):355-374.

5. Vijaimohan K, Jainu M, Sabitha KE, Subramaniyam S, Anandhan C, Shyamala Devi CS: Beneficial effects of alpha linolenic acid rich flaxseed oil on growth performance and hepatic cholesterol metabolism in high fat diet fed rats. Life Sci 2006, 79(5):448-454.

6. Kim HK, Choi H: Dietary alpha-linolenic acid lowers postprandial lipid levels with increase of eicosapentaenoic and docosahexaenoic acid contents in rat hepatic membrane. Lipids 2001, 36(12):1331-1336.

7. Tzang B-S, Yang S-F, Fu S-G, Yang H-C, Sun H-L, Chen Y-C: Effects of dietary flaxseed oil on cholesterol metabolism of hamsters. Food Chem 2009, 114(4):1450-1455.

8. Zhao G, Etherton TD, Martin KR, Gillies PJ, West SG, Kris-Etherton PM: Dietary alpha-linolenic acid inhibits proinflammatory cytokine production by peripheral blood mononuclear cells in hypercholesterolemic subjects. Am J Clin Nutr 2007, 85(2):385-391.

9. Caughey GE, Mantzioris E, Gibson RA, Cleland LG, James MJ: The effect on human tumor necrosis factor alpha and interleukin 1 beta production of diets enriched in n-3 fatty acids from vegetable oil or fish oil. Am J Clin Nutr 1996, 63(1):116-122.

10. Trebušak T, Levart A, Voljč M, Tomažin U, Pirman T: The effect of linseed oil supplementation on performance, fatty acid composition and oxidative status of rabbits. Acta agriculturae Slovenica 2011, 98(2):119-125.

11. Nestel PJ, Pomeroy SE, Sasahara T, Yamashita T, Liang YL, Dart AM, Jennings $\mathrm{GL}$, Abbey M, Cameron JD: Arterial compliance in obese subjects is improved with dietary plant n-3 fatty acid from flaxseed oil despite increased LDL oxidizability. Arterioscler Thromb Vasc Biol 1997, 17(6):1163-1170.

12. Shay KP, Moreau RF, Smith EJ, Smith AR, Hagen TM: Alpha-lipoic acid as a dietary supplement: molecular mechanisms and therapeutic potential. Biochim Biophys Acta 2009, 1790(10):1149-1160.

13. Packer L: Alpha-Lipoic acid: a metabolic antioxidant which regulates NF-kappa B signal transduction and protects against oxidative injury. Drug Metab Rev 1998, 30(2):245-275.

14. Evans JL, Goldfine ID: Alpha-lipoic acid: a multifunctional antioxidant that improves insulin sensitivity in patients with type 2 diabetes. Diabetes technology \& therapeutics 2000, 2(3):401-413.

15. Packer L, Witt EH, Tritschler HJ: Alpha-Lipoic acid as a biological antioxidant. Free Radic Biol Med 1995, 19(2):227-250.

16. Baydas G, Yilmaz O, Celik S, Yasar A, Gursu MF: Effects of certain micronutrients and melatonin on plasma lipid, lipid peroxidation, and homocysteine levels in rats. Arch Med Res 2002, 33(6):515-519.

17. Ford I, Cotter MA, Cameron NE, Greaves M: The effects of treatment with alpha-lipoic acid or evening primrose oil on vascular hemostatic and lipid risk factors, blood flow, and peripheral nerve conduction in the streptozotocin-diabetic rat. Metabolism 2001, 50(8):868-875.

18. Yang RL, Li W, Shi YH, Le GW: Lipoic acid prevents high-fat diet-induced dyslipidemia and oxidative stress: a microarray analysis. Nutrition 2008, 24(6):582-588.

19. Sola S, Mir MQ, Cheema FA, Khan-Merchant N, Menon RG, Parthasarathy S, Khan BV: Irbesartan and lipoic acid improve endothelial function and reduce markers of inflammation in the metabolic syndrome: results of the Irbesartan and Lipoic Acid in Endothelial Dysfunction (ISLAND) study. Circulation 2005, 111(3):343-348.

20. Kono Y: Generation of superoxide radical during autoxidation of hydroxylamine and an assay for superoxide dismutase. Arch Biochem Biophys 1978, 186(1):189-195.

21. Goth $L:$ A simple method for determination of serum catalase activity and revision of reference range. Clin Chim Acta 1991, 196(2-3):143-151.

22. Sazuka Y, Tanizawa H, Takino Y: Effect of adriamycin on the activities of superoxide dismutase, glutathione peroxidase and catalase in tissues of mice. Jpn J Cancer Res 1989, 80(1):89-94.

23. Moron MS, Depierre JW, Mannervik B: Levels of glutathione, glutathione reductase and glutathione S-transferase activities in rat lung and liver. Biochim Biophys Acta 1979, 582(1):67-78.

24. Buege JA, Aust SD: Microsomal lipid peroxidation. Methods Enzymol 1978, 52:302-310 
25. Xu J, Zhou X, Deng Q, Huang Q, Yang J, Huang F: Rapeseed oil fortified with micronutrients reduces atherosclerosis risk factors in rats fed a high-fat diet. Lipids Health Dis 2011, 10:96

26. Lowry OH, Rosebrough NJ, Farr AL, Randall RJ: Protein measurement with the Folin phenol reagent. J Biol Chem 1951, 193(1):265-275.

27. Rodriguez-Leyva D, Dupasquier CM, McCullough R, Pierce GN: The cardiovascular effects of flaxseed and its omega-3 fatty acid, alphalinolenic acid. Can J Cardiol 2010, 26(9):489-496.

28. Wollin SD, Jones PJ: Alpha-lipoic acid and cardiovascular disease. J Nutr 2003, 133(11):3327-3330

29. Madamanchi NR, Vendrov A, Runge MS: Oxidative stress and vascular disease. Arterioscler Thromb Vasc Biol 2005, 25(1):29-38.

30. Bonomini F, Tengattini S, Fabiano A, Bianchi R, Rezzani R: Atherosclerosis and oxidative stress. Histol Histopathol 2008, 23(3):381-390.

31. Formigari A, Irato $P$, Santon A: Zinc, antioxidant systems and metallothionein in metal mediated-apoptosis: biochemical and cytochemical aspects. Comp Biochem Physiol C Toxicol Pharmacol 2007, 146(4):443-459.

32. Dringen R: Metabolism and functions of glutathione in brain. Prog Neurobiol 2000, 62(6):649-671.

33. Moini $H$, Packer L, Saris NE: Antioxidant and prooxidant activities of alpha-lipoic acid and dihydrolipoic acid. Toxicol Appl Pharmacol 2002, 182(1):84-90.

34. Thirunavukkarasu V, Anuradha CV: Influence of alpha-lipoic acid on lipid peroxidation and antioxidant defence system in blood of insulinresistant rats. Diabetes, obesity \& metabolism 2004, 6(3):200-207.

35. Jesudason EP, Masilamoni JG, Jebaraj CE, Paul SF, Jayakumar R: Efficacy of DL-alpha lipoic acid against systemic inflammation-induced mice: antioxidant defense system. Mol Cell Biochem 2008, 313(1-2):113-123.

36. Kim HK, Choi $\mathrm{S}$, Choi $\mathrm{H}$ : Suppression of hepatic fatty acid synthase by feeding alpha-linolenic acid rich perilla oil lowers plasma triacylglycerol level in rats. J Nutr Biochem 2004, 15(8):485-492.

37. Ide T, Kobayashi H, Ashakumary L, Rouyer IA, Takahashi Y, Aoyama T, Hashimoto T, Mizugaki M: Comparative effects of perilla and fish oils on the activity and gene expression of fatty acid oxidation enzymes in rat liver. Biochim Biophys Acta 2000, 1485(1):23-35.

38. Huong DT, Ide T: Dietary lipoic acid-dependent changes in the activity and mRNA levels of hepatic lipogenic enzymes in rats. Br J Nutr 2008, 100(1):79-87.

39. Kabir $Y$, Ide T: Activity of hepatic fatty acid oxidation enzymes in rats fed alpha-linolenic acid. Biochim Biophys Acta 1996, 1304(2):105-119.

40. Libby P: Inflammation in atherosclerosis. Nature 2002, 420(6917):868-874.

41. Willerson JT, Ridker PM: Inflammation as a cardiovascular risk factor. Circulation 2004, 109(21 Suppl 1):|12-II10.

42. Libby P, Ridker PM, Hansson GK: Inflammation in atherosclerosis: from pathophysiology to practice. J Am Coll Cardiol 2009, 54(23):2129-2138.

43. Ridker PM, Rifai N, Rose L, Buring JE, Cook NR: Comparison of C-reactive protein and low-density lipoprotein cholesterol levels in the prediction of first cardiovascular events. N Engl J Med 2002, 347(20):1557-1565.

44. Tzoulaki I, Murray GD, Lee AJ, Rumley A, Lowe GD, Fowkes FG: C-reactive protein, interleukin-6, and soluble adhesion molecules as predictors of progressive peripheral atherosclerosis in the general population: Edinburgh Artery Study. Circulation 2005, 112(7):976-983.

45. Larsson PT, Hallerstam S, Rosfors S, Wallen NH: Circulating markers of inflammation are related to carotid artery atherosclerosis. Int Angiol 2005, 24(1):43-51.

46. Rallidis LS, Paschos G, Liakos GK, Velissaridou AH, Anastasiadis G, Zampelas A: Dietary alpha-linolenic acid decreases $C$-reactive protein, serum amyloid A and interleukin-6 in dyslipidaemic patients. Atherosclerosis 2003, 167(2):237-242.

47. Ying Z, Kampfrath T, Sun Q, Parthasarathy S, Rajagopalan S: Evidence that alpha-lipoic acid inhibits NF-kappaB activation independent of its antioxidant function. Inflamm Res 2011, 60(3):219-225.

48. Lee HA, Hughes DA: Alpha-lipoic acid modulates NF-kappaB activity in human monocytic cells by direct interaction with DNA. Exp Gerontol 2002, 37(2-3):401-410.

doi:10.1186/1476-511X-11-148

Cite this article as: Xu et al:: Flaxseed oil and a-lipoic acid combination reduces atherosclerosis risk factors in rats fed a high-fat diet. Lipids in Health and Disease 2012 11:148.

\section{Submit your next manuscript to BioMed Central and take full advantage of:}

- Convenient online submission

- Thorough peer review

- No space constraints or color figure charges

- Immediate publication on acceptance

- Inclusion in PubMed, CAS, Scopus and Google Scholar

- Research which is freely available for redistribution 